Trab-813-02 (8 páginas)

\title{
INFLUÊNCIA DE DOSES CRESCENTES DE CHUMBO SOBRE O TEOR E O CONTEÚDO DE NUTRIENTES E Pb EM MUDAS DE IPÊ-ROXO (Tabebuia impetiginosa (Mart.) Standl.) ${ }^{1}$
}

\author{
Haroldo Nogueira de Paiva ${ }^{2}$, Janice Guedes de Carvalho ${ }^{3}$, José Oswaldo Siqueira ${ }^{3}$, Antônio Rodrigues Fernandes ${ }^{4}$ e \\ José Romilson Paes de Miranda ${ }^{5}$
}

\begin{abstract}
RESUMO - O efeito da aplicação de chumbo sobre o teor e o conteúdo de nutrientes e $\mathrm{Pb}$ na raiz, no caule e nas folhas de mudas de ipê-roxo (Tabebuia impetiginosa (Mart.) Standl.) foi estudado em um experimento realizado em casa de vegetação do Departamento de Ciência do Solo da Universidade Federal de Lavras. As mudas foram cultivadas em solução nutritiva de Clark e submetidas a doses crescentes de $\mathrm{Pb}: 0,48,96,192$ e $288 \mu \mathrm{mol} / \mathrm{l}$. O experimento foi conduzido em delineamento estatístico de blocos ao acaso, e após 60 dias de exposição ao metal pesado foram feitas avaliações do teor e do conteúdo de macro, micronutrientes e chumbo na matéria seca de raiz, caule e folha. Os resultados mostraram que a aplicação de chumbo aumentou o teor de $\mathrm{P}$ e praticamente não afetou os teores de $\mathrm{S}, \mathrm{Ca}$ e $\mathrm{Mg}$ nas mudas. De modo geral os teores de $\mathrm{Cu}, \mathrm{Fe}$ e $\mathrm{Mn}$ sofreram redução, o teor de $\mathrm{Zn}$ não foi afetado, enquanto o conteúdo de macro e de micronutrientes sofreu redução. $\mathrm{O}$ teor de $\mathrm{Pb}$ na matéria seca de raiz, caule e folha aumentou com as doses aplicadas, principalmente na raiz, e se mostrou pouco móvel nas plantas analisadas.
\end{abstract}

Palavras-chave: Espécie florestal nativa, metal pesado, nutrição de plantas e toxidez.

\section{INFLUENCE OF INCREASING LEAD LEVELS ON NUTRIENT AND Pb CONTENT AND ACCUMULATION IN IPE-ROXO (Tabebuia impetiginosa (Mart.) Standl.) SEEDLINGS}

\begin{abstract}
The effect of lead application on nutrient and Pb content and accumulation in ipê-roxo (Tabebuia impetiginosa (Mart.) Standl.) seedlings was studied in an experiment conducted under greenhouse conditions at the Soil Science Department of the Federal University of Lavras. The seedlings were cultivated in Clark nutrient solution and submitted to increasing Pb levels: 0, 48, 96, 192 and $288 \mu \mathrm{mol} / \mathrm{l}$. The experiment was arranged in a randomized block design and after 60 days of exposure to the heavy metals, evaluations of macro and micronutrient and lead content and accumulation in the root, stem and leaf dry matter were made. The results showed that lead application increased P content and practically did not affect $S, C a$ and $\mathrm{Mg}$ contents. $\mathrm{Cu}, \mathrm{Fe}$ and $\mathrm{Mn}$ contents, in general, presented a reduction; Zn content was not affected while macro and micronutrient accumulation was reduced. Lead content and accumulation in the root, stem and leaf dry matter increased with the doses applied.
\end{abstract}

Key words: $\quad$ Native forest species, heavy metal, plant nutrition and toxicity.

\section{INTRODUÇÃO}

A forma de distribuição de $\mathrm{Pb}$ nas plantas é muito variável com a espécie, dependendo principalmente das condições ambientais em que a planta está inserida.
Portanto, é de se esperar que haja maior acúmulo nas raízes de plantas que crescem em solos ou soluções contaminadas, mas espera-se que haja maior acúmulo na parte aérea de plantas que estão submetidas à poluição do ar contaminado por este metal.

1 Recebido para publicação em 29.1.2002.

Aceito para publicação em 19.2.2003.

Parte da tese apresentada à Universidade Federal de Lavras pelo primeiro autor.

2 Departamento de Engenharia Florestal da Universidade Federal de Viçosa - UFV, 36571-000 Viçosa-MG, $<$ hnpaiva@mail.ufv.br>. ${ }^{3}$ Departamento de Ciência do Solo da Universidade Federal de Lavras - UFLA, 37200-000 Lavras-MG.

${ }^{4}$ Departamento de Solos da Universidade Federal Rural da Amazônia - UFRA, 66077-530 Belém-PA. ${ }^{5}$ Departamento de Engenharia Florestal da Universidade Federal da Paraíba - UFPB, 58700-970 Patos-PB. 
A absorção de $\mathrm{Pb}$ dá-se por mecanismo passivo, sendo ele absorvido por pêlos radiculares e consideravelmente armazenado na parede celular (Kabata-Pendias \& Pendias, 1984). A absorção de nutrientes é definida como sendo o processo pelo qual o elemento (nutriente) passa do substrato (solo, solução nutritiva) para uma parte qualquer da célula (parede, citoplasma e vacúolo) (Malavolta et al., 1997).

De acordo com Kabata-Pendias \& Pendias (1984), o teor de $\mathrm{Pb}$ em plantas que crescem sobre áreas de mineração é geralmente correlacionado com a concentração $\mathrm{de} \mathrm{Pb}$ no solo, embora esta relação seja diferente entre os órgãos da planta. Bharti \& Singh (1993) e Kastori et al. (1998) constataram que plantas que crescem em solução nutritiva apresentam absorção positivamente correlacionada com a dose de $\mathrm{Pb}$ aplicada

De acordo com Baumhardt \& Welch (1972), plantas de milho absorvem e translocam $\mathrm{Pb}$ para a parte aérea, mas este não é redistribuído para os grãos. Em raiz, caule e folha de gergelim, o acúmulo de $\mathrm{Pb}$ aumentou com as doses do metal, que variaram de 10 a $2.000 \mu \mathrm{mol}$, mas este acúmulo foi consideravelmente maior nas raízes que nas folhas das mudas, mostrando que ocorre baixa translocação do metal para as folhas (Bharti \& Singh, 1993). Em Picea abies, o teor de $\mathrm{Pb}$ nas raízes foi cerca de dez vezes maior que no caule (Marschner et al., 1996).

Em termos nutricionais, Huang \& Cunningham (1996) constataram que o $\mathrm{Pb}$ inibiu a concentração de cálcio nas raízes, e que na parte aérea houve redução (40\%) na concentração de $\mathrm{Ca}$ em milho, mas nenhum efeito foi verificado em Ambrosia artemisiifolia. Na parte aérea, a concentração de $\mathrm{Mg}, \mathrm{K}, \mathrm{Fe}, \mathrm{Mn}$ e $\mathrm{Zn}$ foi reduzida significativamente nas duas espécies, após duas semanas de exposição a $20 \mu \mathrm{mol} \mathrm{Pb}$. A concentração de $\mathrm{P}$ nas raízes de Ambrosia artemisiifolia aumentou com a aplicação de $\mathrm{Pb}$, porém o mesmo tratamento não afetou significativamente a concentração em milho. Quanto ao $\mathrm{P}$ na parte aérea, não houve efeito do tratamento com $\mathrm{Pb}$.

Para Eucalyptus maculata e Eucalyptus urophylla, Soares (1999) verificou comportamento distinto entre as espécies. Houve acréscimo na concentração de $\mathrm{Cu}$ na parte aérea de E. urophylla e redução em E. maculata, a concentração de $\mathrm{Zn}$ e Fe foi pouco influenciada pelas doses de $\mathrm{Pb}$ e a de $\mathrm{Mn}$ teve resposta tipo raiz quadrática. Nas raízes, a concentração de $\mathrm{Cu}, \mathrm{Zn}$ e $\mathrm{Mn}$ não diferiu significativamente, ao passo que a concentração de $\mathrm{Fe}$

R. Árvore, Viçosa-MG, v.27, n.2, p.151-158, 2003 aumentou bastante para E. urophylla. Para os macronutrientes, o autor constatou comportamento não consistente para concentração de $\mathrm{P}$, porém não observou diferença significativa sobre a concentração de $\mathrm{K}$ e de $\mathrm{Mg}$, ao passo que houve resposta quadrática para $\mathrm{Se} \mathrm{Ca}$, na parte aérea. Nas raízes, houve aumento na concentração de $\mathrm{P}$, redução na concentração de $\mathrm{S}, \mathrm{Ca}$ e $\mathrm{Mg}$ e nenhum efeito sobre o K.

Os estudos sobre o comportamento de espécies lenhosas em ambientes contaminados com metais pesados são relativamente escassos, o que pode ser atribuído à menor tolerância destas espécies quando comparadas com espécies herbáceas e gramíneas (Eltrop et al., 1991). Considerando o fato de que grande parte das áreas de mineração são multicontaminadas por metais pesados, dentre eles o Pb, é importante que sejam feitos estudos com o intuito de conhecer a adaptação de espécies lenhosas nestes ambientes.

O objetivo deste trabalho foi verificar o efeito de doses crescentes de $\mathrm{Pb}$, em solução nutritiva, sobre o teor e o conteúdo de macro e micronutrientes e de $\mathrm{Pb}$, em diferentes partes de mudas de ipê-roxo.

\section{MATERIAL E MÉTODOS}

O experimento foi conduzido em casa de vegetação do Departamento de Ciência do Solo da Universidade Federal de Lavras - UFLA, utilizando mudas de ipê-roxo (Tabebuia impetiginosa (Mart.) Standl.).

As mudas foram produzidas em substrato que continha areia lavada, e quando apresentavam altura média de $5 \mathrm{~cm}$ ou dois pares de folhas definitivas foram repicadas para bandejas plásticas com capacidade de 35 litros, contendo solução nutritiva de Clark (Clark, 1975). A concentração de todos os nutrientes estava reduzida a 30\%, com aeração constante. As mudas permaneceram neste ambiente por 15 dias, quando a solução foi substituída e a concentração de todos os nutrientes foi elevada para $50 \%$ da normal. Ao final de 15 dias as mudas foram individualizadas em vasos plásticos, com capacidade de $900 \mathrm{ml}$, contendo solução nutritiva normal, sendo trocada a cada dez dias. Após 40 dias, foram aplicadas doses crescentes de chumbo, ajustando-se o $\mathrm{pH}$ para 5,5.

Os tratamentos consistiram na aplicação de doses crescentes de chumbo: 0, 48, 96, 192 e $288 \mu \mathrm{mol} / 1$, usando como fonte o acetato de chumbo $\left[\mathrm{Pb}\left(\mathrm{CH}_{3} \mathrm{COO}\right)_{2}\right.$. 
$\left.3 \mathrm{H}_{2} \mathrm{O}\right]$. Na preparação de todas as soluções-estoque dos nutrientes e do chumbo, empregaram-se reagentes PA. A solução nutritiva foi preparada com água deionizada, e durante o intervalo de renovação da solução o volume dos vasos foi completado, sempre que necessário, também com água deionizada.

As plantas permaneceram em exposição ao metal pesado por 60 dias, com renovação da solução nutritiva a cada dez dias, mantendo-se o $\mathrm{pH}$ em 5,5 pela adição de $\mathrm{NaOH}$ ou $\mathrm{HCl} 0,1 \mathrm{~mol} / 1$. Após este período procedeu-se à colheita das plantas, separando-as em raiz, caule e folhas.

As raízes, o caule e as folhas foram lavadas em água destilada e secas em estufa com circulação de ar a $65^{\circ} \mathrm{C}$, até peso constante. $\mathrm{O}$ peso de matéria seca foi determinado em balança de precisão $(0,01 \mathrm{~g})$ e em seguida foi passada em moinho tipo Wiley, equipado com peneira de $0,38 \mathrm{~mm}$, para ser analisada quimicamente.

Após a digestão nitricoperclórica, os teores de $\mathrm{Ca}$, $\mathrm{Mg}, \mathrm{Fe}, \mathrm{Cu}, \mathrm{Mn}, \mathrm{Zn}$ e $\mathrm{Pb}$ na raiz, no caule e nas folhas foram determinados por espectrofotometria de absorção atômica. Os teores de $\mathrm{P}$ foram determinados por colorimetria, os teores de S por turbidimetria e os de K por fotometria de chama (Malavolta et al., 1997).

Os conteúdos de $\mathrm{P}, \mathrm{K}, \mathrm{S}, \mathrm{Ca}, \mathrm{Mg}, \mathrm{Fe}, \mathrm{Cu}, \mathrm{Mn}, \mathrm{Zn}$ e $\mathrm{Pb}$ na raiz, no caule, nas folhas, na parte aérea e total foram calculados com base nos teores e nas produções de matéria seca.

O delineamento estatístico adotado foi o de blocos ao acaso, com cinco tratamentos e cinco repetições, sendo cada repetição representada por um vaso com uma planta, perfazendo, assim, um total de 25 plantas.

Os dados foram submetidos à análise de variância, e foram ajustadas equações de regressão entre as doses de chumbo aplicadas e o teor e o conteúdo dos diferentes nutrientes analisados.

\section{RESULTADOS E DISCUSSÃO}

As equações de regressão ajustadas para os teores de macronutrientes na raiz, no caule e nas folhas das mudas de ipê-roxo, em função das doses de chumbo aplicadas em solução nutritiva, mostram que este metal pesado afeta tais teores de forma diferenciada com a parte da planta e com o nutriente (Quadro 1).

Quadro 1 - Equações de regressão para os teores de macronutrientes na raiz, no caule e nas folhas de mudas de ipê-roxo, em resposta a doses crescentes de chumbo

Table 1 - Regression equations of macronutrients content in the root, stem and leaves of ipê-roxo seedlings, in response to increasing levels of lead

\begin{tabular}{|c|c|c|c|}
\hline Nutriente & Parte da Planta & Equação de regressão & $\mathrm{R}^{2}$ \\
\hline \multirow{3}{*}{$\mathrm{P}$} & Raiz & $\mathrm{Y}=2,3+0,0077 * \mathrm{X}$ & 0,95 \\
\hline & Caule & $\mathrm{Y}=2,0+0,0107 * * \mathrm{X}-0,000030 * * \mathrm{X}^{2}$ & 0,81 \\
\hline & Folha & $\mathrm{Y}=1,6-0,0049^{* *} \mathrm{X}+0,000016^{* *} \mathrm{X}^{2}$ & 0,95 \\
\hline \multirow{3}{*}{$\mathrm{K}$} & Raiz & $\mathrm{Y}=2,3+0,0242 * * \mathrm{X}-0,000092 * * \mathrm{X}^{2}$ & 0,91 \\
\hline & Caule & $\mathrm{Y}=10,6-0,0143 * * \mathrm{X}$ & 0,85 \\
\hline & Folha & $\mathrm{Y}=15,7$ & \\
\hline \multirow{3}{*}{$\mathrm{S}$} & Raiz & $\mathrm{Y}=2,8$ & \\
\hline & Caule & $\mathrm{Y}=0,8$ & \\
\hline & Folha & $\mathrm{Y}=1,4-0,0021 * * \mathrm{X}+0,000006 * * \mathrm{X}^{2}$ & 0,61 \\
\hline \multirow{3}{*}{$\mathrm{Ca}$} & Raiz & $\mathrm{Y}=1,6$ & \\
\hline & Caule & $\mathrm{Y}=6,0$ & \\
\hline & Folha & $\mathrm{Y}=9,9+0,0308 * * \mathrm{X}-0,000072 * * \mathrm{X}^{2}$ & 0,91 \\
\hline \multirow{3}{*}{$\mathrm{Mg}$} & Raiz & $\mathrm{Y}=0,4$ & \\
\hline & Caule & $Y=1,4+0,0055^{* *} \mathrm{X}-0,000018 * * \mathrm{X}^{2}$ & 0,88 \\
\hline & Folha & $\mathrm{Y}=2,9$ & \\
\hline
\end{tabular}

** significativo a $1 \%$ de probabilidade, pelo teste $\mathrm{F}$. 
A presença de chumbo em solução nutritiva induziu ao aumento linear no teor radicular de P, embora KabataPendias \& Pendias (1984) preconizem que a presença de $\mathrm{Pb}$ provoca antagonismo sobre a absorção de $\mathrm{P}$.

Esse aumento no teor radicular de P pode ser explicado pela precipitação do fósforo na forma de fosfato de chumbo, o que, segundo Kabata-Pendias \& Pendias (1984), é esperado. Esta mesma tendência foi observada em espécies de eucalipto (Soares, 1999) e em Ambrosia artemisiifolia (Huang \& Cunningham, 1996).

No caule das mudas de ipê-roxo o teor de P apresentou resposta quadrática negativa, enquanto o teor foliar de $\mathrm{P}$ apresentou resposta quadrática positiva. No presente trabalho, as doses de chumbo aplicadas não chegaram a comprometer o teor de $\mathrm{P}$ nas plantas, pois, de acordo com Bergmann (1992), teores de P que variam de 1 a $5 \mathrm{~g} / \mathrm{kg}$ são requeridos para o ótimo crescimento de plantas.

O teor de $\mathrm{K}$ na raiz aumentou até a dose $131,5 \mu \mathrm{mol}$ $\mathrm{Pb}$, reduzindo a partir de então, enquanto o teor caulinar sofreu redução linear e o teor foliar não apresentou efeito significativo. Esta redução no teor radicular de K pode, possivelmente, ser explicada pela inibição competitiva entre o $\mathrm{K}$ e os cátions divalentes presentes em altas concentrações (Walker et al., 1977). De acordo com Faquin (1994) e Malavolta et al. (1997), a presença de $\mathrm{Mg}^{+2}$ e $\mathrm{Ca}^{+2}$, em altas concentrações, promove inibição competitiva com a absorção de $\mathrm{K}^{+}$. A ausência de efeito significativo sobre o teor de $\mathrm{K}$ nas folhas está de acordo com os resultados obtidos por Simon (1998), Lagriffoul et al. (1998) e Soares (1999), em que a concentração de $\mathrm{K}$ na parte aérea não foi afetada pelo aumento das doses de metais pesados, o que, provavelmente, se deve ao fato de o K ser um cátion, a exemplo dos metais pesados.

Não houve efeito significativo no teor de $\mathrm{S}$ na raiz e no caule das mudas de ipê-roxo com a aplicação de $\mathrm{Pb}$, enquanto nas folhas apresentou resposta quadrática positiva, com redução neste teor até a dose $175 \mu \mathrm{mol}$ $\mathrm{Pb}$, sendo crescente a partir de então. Resultados semelhantes foram observados em Eucalyptus urophylla e Eucalyptus maculata (Soares, 1999), o que era esperado, pois de acordo com Kabata-Pendias \& Pendias (1984) a presença de metais pesados, como o $\mathrm{Pb}$, não exerce qualquer efeito sobre a absorção de $\mathrm{S}$.

O teor de $\mathrm{Ca}$ na raiz e no caule de mudas de ipêroxo não foi afetado pelas doses de $\mathrm{Pb}$ aplicadas, ao passo que nas folhas houve resposta quadrática negativa com a aplicação de $\mathrm{Pb}$. Houve aumento no teor foliar de Ca até a dose $214 \mu \mathrm{mol} \mathrm{Pb}$, caindo a partir desta dose. Quanto ao teor de Mg, observa-se, no Quadro 1, que o seu teor na raiz e nas folhas não apresentou efeito significativo quando da aplicação de $\mathrm{Pb}$. Já o teor caulinar de $\mathrm{Mg}$ apresentou resposta quadrática negativa com a presença de $\mathrm{Pb}$, aumentando até a dose $153 \mu$ mol $\mathrm{Pb}$, quando alcança um teor de $1,82 \mathrm{~g} / \mathrm{kg}$. Na maior dose aplicada $(288 \mu \mathrm{mol} \mathrm{Pb})$ este teor chegou a $1,49 \mathrm{~g} / \mathrm{kg}$, o que está dentro dos limites normais em plantas, que são de 1 a $5 \mathrm{~g} / \mathrm{kg}$ (Bergmann, 1992).

$\mathrm{O}$ teor de $\mathrm{Pb}$ e de alguns micronutrientes na matéria seca de raiz, caule e folhas de mudas de ipê-roxo foi significativamente afetado pela aplicação de doses crescentes $\mathrm{de} \mathrm{Pb}$, em solução nutritiva, apresentando resposta diferenciada com a parte da planta e com o nutriente analisado (Quadro 2).

Na raiz, a aplicação de $\mathrm{Pb}$ não exerceu efeito significativo sobre o teor de $\mathrm{Cu}$. $\mathrm{O} \mathrm{Pb}$ é considerado um elemento que não interfere na absorção de $\mathrm{Cu}$ (KabataPendias e Pendias, 1984), resultando em sua não-significância sobre o teor de Cu na raiz de ipê-roxo (Quadro 2). No caule, a presença de $\mathrm{Pb}$ fez com que o teor de $\mathrm{Cu}$ apresentasse resposta quadrática positiva, alcançando o teor mínimo quando da aplicação de $165 \mu \mathrm{mol} \mathrm{Pb}$. O Pb reduziu linearmente o teor foliar de $\mathrm{Cu}$, mostrando que este elemento, apesar de não interferir na absorção de Cu (Kabata-Pendias \& Pendias, 1984), afeta o seu transporte para a parte aérea, a exemplo do que ocorre com a aplicação de Cd (Obata \& Umebayashi, 1997) e de Ni (Yang et al., 1996).

O teor de ferro na raiz das mudas de ipê-roxo aumentou de forma linear com a aplicação de $\mathrm{Pb}$. No caule e nas folhas, o ferro diminui até determinada dose, passando a apresentar um teor crescente com doses mais elevadas de $\mathrm{Pb}$, o que indica que até certa dose de $\mathrm{Pb}$ há restrição do transporte de Fe da raiz para a parte aérea, conforme observado em várias espécies por Yang et al. (1996).

Nas folhas das mudas de ipê-roxo o teor de Mn apresentou resposta quadrática positiva com a aplicação de doses crescentes de $\mathrm{Pb}$, alcançando teor mínimo quando da aplicação de $280 \mu \mathrm{mol} \mathrm{Pb}$. Quanto ao teor radicular e caulinar de Mn, observa-se, no Quadro 2, que a aplicação de $\mathrm{Pb}$ não exerceu efeito significativo. $\mathrm{O}$ teor de Zn, nas diferentes partes analisadas das plantas, não apresentou resposta significativa quando da aplicação de $\mathrm{Pb}$. 
Quadro 2 - Equações de regressão para os teores de micronutrientes e de chumbo na raiz, no caule e nas folhas de mudas de ipê-roxo, em resposta a doses crescentes de chumbo

Table 2 - Regression equations of micronutrients and lead content in the root, stem and leaves of ipê-roxo seedlings in response to increasing levels of lead

\begin{tabular}{|c|c|c|c|}
\hline Nutriente & Parte da Planta & Equação de Regressão & $\mathrm{R}^{2}$ \\
\hline \multirow{3}{*}{$\mathrm{Cu}$} & Raiz & $\mathrm{Y}=23,1$ & \\
\hline & Caule & $\mathrm{Y}=5,5-0,0415^{* *} \mathrm{X}+0,000126^{* *} \mathrm{X}^{2}$ & 0,74 \\
\hline & Folha & $\mathrm{Y}=4,1-0,0061 * * \mathrm{X}$ & 0,81 \\
\hline \multirow{3}{*}{$\mathrm{Fe}$} & Raiz & $\mathrm{Y}=241+1,7417 * * \mathrm{X}$ & 0,94 \\
\hline & Caule & $Y=97,8-7,6010 * * X^{0,5}+0,4169 * * X$ & 0,84 \\
\hline & Folha & $\mathrm{Y}=135-0,4050^{* *} \mathrm{X}+0,001225^{*} \mathrm{X}^{2}$ & 0,81 \\
\hline \multirow{3}{*}{$\mathrm{Mn}$} & Raiz & $\mathrm{Y}=32,0$ & \\
\hline & Caule & $\mathrm{Y}=17,5$ & \\
\hline & Folha & $Y=105-0,5739 * * X+0,001556 * X^{2}$ & 0,79 \\
\hline \multirow{3}{*}{$\mathrm{Zn}$} & Raiz & $\mathrm{Y}=62,0$ & \\
\hline & Caule & $\mathrm{Y}=79,0$ & \\
\hline & Folha & $\mathrm{Y}=65,0$ & \\
\hline \multirow{3}{*}{$\mathrm{Pb}$} & Raiz & $\mathrm{Y}=3496+134,43 * * \mathrm{X}$ & 0,89 \\
\hline & Caule & $\mathrm{Y}=99+1,2943 * * \mathrm{X}$ & 0,96 \\
\hline & Folha & $\mathrm{Y}=6,9+0,6249^{* *} \mathrm{X}-0,001461^{* *} \mathrm{X}^{2}$ & 0,92 \\
\hline
\end{tabular}

** significativo a $1 \%$ de probabilidade, pelo teste $\mathrm{F}$.

Nas mudas de ipê-roxo o teor de $\mathrm{Pb}$ apresentou aumento linear na raiz e no caule, enquanto na folha a resposta foi quadrática negativa, com o máximo de $73,7 \mathrm{mg} / \mathrm{kg}$, na dose $213,8 \mu \mathrm{mol} \mathrm{Pb}$, valor este $968 \%$ superior ao do tratamento-controle. Constatou-se que o teor de $\mathrm{Pb}$ é muito superior na raiz, quando comparado com o do caule e o da folha, confirmando resultados obtidos por diferentes autores (Bharti \& Singh, 1993; Huang \& Cunningham, 1996; Marschner et al., 1996; Kastori et al., 1998; Fodor et al., 1998). O teor radicular foi 86 vezes superior ao teor caulinar e 437 vezes superior ao teor foliar (no ponto de máximo teor foliar), refletindo, de forma clara, a concentração na raiz e sua baixa mobilidade nas plantas.

O conteúdo de macronutrientes foi afetado pela aplicação de doses crescentes de $\mathrm{Pb}$ (Quadro 3), observando-se, de modo geral, redução no conteúdo destes. $\mathrm{O}$ conteúdo radicular de $\mathrm{P}$ não apresentou efeito significativo da aplicação de $\mathrm{Pb}$, no entanto todas as demais partes da planta analisadas apresentaram resposta quadrática positiva, mostrando que há redução no conteúdo até determinada dose de $\mathrm{Pb}$, passando este conteúdo a ser crescente a partir de então. O conteúdo de $\mathrm{P}$ apresentou pontos de mínimo quando da aplicação de 197, 216, 210 e $223 \mu \mathrm{mol} \mathrm{Pb}$ para o caule, a folha, a parte aérea e total, respectivamente. Essa redução no conteúdo pode ser justificada pela redução na produção de matéria seca, conforme observado por Paiva et al. (2000), em mudas de ipê-roxo.

O K apresentou respostas parecidas com o conteúdo de P. Portanto, o conteúdo radicular não foi afetado de forma significativa, enquanto o conteúdo nas folhas, na parte aérea e total mostrou redução linear com a aplicação de $\mathrm{Pb}$. O conteúdo de $\mathrm{S}$, a exemplo do que ocorreu com o conteúdo de $\mathrm{P}$ e de $\mathrm{K}$ na raiz, não apresentou resposta significativa, mas no caule, na folha e na parte aérea a resposta foi quadrática positiva, alcançando o mínimo quando da aplicação de 216, 231 e $229 \mu \mathrm{mol} \mathrm{Pb}$, respectivamente. $\mathrm{O}$ conteúdo total de $\mathrm{S}$ diminuiu linearmente com as doses de $\mathrm{Pb}$.

Cálcio e magnésio, em mudas de ipê-roxo, apresentaram redução linear em seus conteúdos nas diferentes partes das plantas analisadas, com exceção no conteúdo caulinar de $\mathrm{Ca}$, cuja resposta foi quadrática positiva. De modo geral, metais pesados, como o $\mathrm{Pb}$, reduzem o conteúdo de $\mathrm{Ca}$ e de $\mathrm{Mg}$ em diferentes espécies de plantas

R. Árvore, Viçosa-MG, v.27, n.2, p.151-158, 2003 
(Huang \& Cunningham, 1996; Soares, 1999), mostrando haver antagonismo entre estes elementos (Kabata-Pendias e Pendias, 1984).

Assim como aconteceu para os macronutrientes, o conteúdo de micronutrientes, nas diferentes partes das mudas de ipê-roxo, foi afetado pelas doses crescentes de $\mathrm{Pb}$ em solução nutritiva, exceção para o conteúdo radicular de $\mathrm{Cu}$, Fe e Zn (Quadro 4).

$\mathrm{O}$ conteúdo radicular de $\mathrm{Cu}$ e de $\mathrm{Fe}$ não foi significativamente afetado pelas doses de $\mathrm{Pb}$, no entanto o conteúdo total de $\mathrm{Cu}$ reduziu-se linearmente e o conteúdo total de Fe apresentou resposta quadrática positiva à aplicação de $\mathrm{Pb}$. Redução no conteúdo de $\mathrm{Cu}$ e de $\mathrm{Fe}$ também foi observada em mudas de Eucalyptus urophylla e Eucalyptus maculata, cultivadas em solução nutritiva contaminada com Pb (Soares, 1999).

O conteúdo de Mn diminuiu de forma linear com a aplicação de $\mathrm{Pb}$, ao passo que o conteúdo de $\mathrm{Zn}$ apresentou resposta quadrática positiva nas diferentes partes das plantas, exceção para o conteúdo radicular de $\mathrm{Zn}$, que não foi significativamente afetado pela aplicação de $\mathrm{Pb}$. No caule e na folha, o conteúdo de Zn atingiu o mínimo quando da aplicação de 239 e $235 \mu \mathrm{mol} \mathrm{Pb}$, respectivamente.

Quadro 3 - Equações de regressão para o conteúdo de macronutrientes em diferentes partes de mudas de ipê-roxo submetidas a doses crescentes de chumbo

Table 3 - Regression equations of macronutrient accumulation in different parts of the ipe-roxo seedlings, in response to increasing levels of lead

\begin{tabular}{|c|c|c|c|}
\hline Nutriente & Parte da Planta & Equação de Regressão & $\mathrm{R}^{2}$ \\
\hline \multirow{5}{*}{$\mathrm{P}$} & Raiz & $\mathrm{Y}=2,8$ & \\
\hline & Caule & $\mathrm{Y}=2,4-0,0201 * * \mathrm{X}+0,000051 * \mathrm{X}^{2}$ & 0,84 \\
\hline & Folha & $\mathrm{Y}=8,3-0,0589 * * \mathrm{X}+0,000136 * * \mathrm{X}^{2}$ & 0,97 \\
\hline & Parte Aérea & $Y=10,7-0,0790 * * X+0,000188^{* *} X^{2}$ & 0,95 \\
\hline & Total & $Y=13,3-0,0727 * * X+0,000163 * * X^{2}$ & 0,93 \\
\hline \multirow{5}{*}{$\mathrm{K}$} & Raiz & $\mathrm{Y}=3,0$ & \\
\hline & Caule & $\mathrm{Y}=13,2-0,0858 * * \mathrm{X}+0,000187 * * \mathrm{X}^{2}$ & 0,97 \\
\hline & Folha & $Y=70,6-0,1704 * * X$ & 0,93 \\
\hline & Parte Aérea & $\mathrm{Y}=81,9-0,2017 * * \mathrm{X}$ & 0,91 \\
\hline & Total & $\mathrm{Y}=86,2-0,2109^{* *} \mathrm{X}$ & 0,93 \\
\hline \multirow{5}{*}{$\mathrm{S}$} & Raiz & $Y=2,6$ & \\
\hline & Caule & $\mathrm{Y}=1,1-0,0069^{* *} \mathrm{X}+0,000016^{* *} \mathrm{X}^{2}$ & 0,95 \\
\hline & Folha & $Y=7,4-0,0471 * * X+0,000102 * * X^{2}$ & 0,98 \\
\hline & Parte Aérea & $\mathrm{Y}=8,4-0,0540 * * \mathrm{X}+0,000118 * \mathrm{X}^{2}$ & 0,98 \\
\hline & Total & $\mathrm{Y}=10,4-0,0244 * * \mathrm{X}$ & 0,87 \\
\hline \multirow{5}{*}{$\mathrm{Ca}$} & Raiz & $Y=1,9-0,0039 * * X$ & 0,92 \\
\hline & Caule & $Y=7,5-0,0428 * * X+0,000097 * * X^{2}$ & 0,89 \\
\hline & Folha & $Y=50,5-0,1105 * * X$ & 0,88 \\
\hline & Parte Aérea & $Y=57,0-0,1249 * * X$ & 0,88 \\
\hline & Total & $\mathrm{Y}=58,9-0,1288 * * \mathrm{X}$ & 0,88 \\
\hline \multirow{5}{*}{$\mathrm{Mg}$} & Raiz & $\mathrm{Y}=0,5-0,0008 * * \mathrm{X}$ & 0,92 \\
\hline & Caule & $\mathrm{Y}=1,6-0,0029 * * \mathrm{X}$ & 0,81 \\
\hline & Folha & $\mathrm{Y}=14,0-0,0371 * * \mathrm{X}$ & 0,80 \\
\hline & Parte Aérea & $\mathrm{Y}=15,6-0,0400 * * \mathrm{X}$ & 0,83 \\
\hline & Total & $Y=16,0-0,0409 * * X$ & 0,84 \\
\hline
\end{tabular}

** significativo a $1 \%$ de probabilidade, pelo teste $\mathrm{F}$.

R. Árvore, Viçosa-MG, v.27, n.2, p.151-158, 2003 
Influência de Doses Crescentes de Chumbo sobre o Teor e ...

Quadro 4 - Equações de regressão para o conteúdo de micronutrientes e de chumbo em diferentes partes das mudas de ipê-roxo submetidas a doses crescentes de chumbo

Table 4 - Regression equations of micronutrient and lead accumulation in different parts of ipê-roxo seedlings, in response to increasing levels of lead

\begin{tabular}{|c|c|c|c|}
\hline Nutriente & Parte da Planta & Equação de Regressão & $\mathrm{R}^{2}$ \\
\hline \multirow{5}{*}{$\mathrm{Cu}$} & Raiz & $Y=19,0$ & \\
\hline & Caule & $Y=7,0-0,0681 * * X+0,000176^{* *} X^{2}$ & 0,77 \\
\hline & Folha & $\mathrm{Y}=18,0-0,0557 * * \mathrm{X}$ & 0,79 \\
\hline & Parte Aérea & $\mathrm{Y}=27,0-0,1733^{* *} \mathrm{X}+0,000346^{* *} \mathrm{X}^{2}$ & 0,93 \\
\hline & Total & $\mathrm{Y}=46,0-0,0967 * \mathrm{X}$ & 0,87 \\
\hline \multirow{5}{*}{$\mathrm{Fe}$} & Raiz & $\mathrm{Y}=364,0$ & \\
\hline & Caule & $Y=109,0-0,8396^{* *} \mathrm{X}+0,002208^{* *} \mathrm{X}^{2}$ & 0,74 \\
\hline & Folha & $Y=721,0-5,1655^{* *} \mathrm{X}+0,011840 * * \mathrm{X}^{2}$ & 0,94 \\
\hline & Parte Aérea & $\mathrm{Y}=830,0-6,0050 * * \mathrm{X}+0,014048 * * \mathrm{X}^{2}$ & 0,93 \\
\hline & Total & $\mathrm{Y}=1124,0-5,1155 * * X+0,012498 * * \mathrm{X}^{2}$ & $0,8 \overline{6}$ \\
\hline \multirow{5}{*}{ Mn } & Raiz & $\mathrm{Y}=39,0-0,0875 * \mathrm{X}$ & 0,83 \\
\hline & Caule & $Y=17,0-0,0278 * * X$ & 0,73 \\
\hline & Folha & $\mathrm{Y}=568,0-1,1668 * * \mathrm{X}$ & 0,94 \\
\hline & Parte Aérea & $\mathrm{Y}=585,0-1,1947 * * \mathrm{X}$ & 0,94 \\
\hline & Total & $\mathrm{Y}=625,0-1,2822 * * \mathrm{X}$ & 0,93 \\
\hline \multirow{5}{*}{$\mathrm{Zn}$} & Raiz & $\mathrm{Y}=56,0$ & \\
\hline & Caule & $\mathrm{Y}=96,0-0,5310 * * \mathrm{X}+0,001201 * * \mathrm{X}^{2}$ & 0,96 \\
\hline & Folha & $\mathrm{Y}=357,0-2,0685 * * \mathrm{X}+0,004321 * * \mathrm{X}^{2}$ & 0,96 \\
\hline & Parte Aérea & $Y=454,0-2,5995 * x+0,005522 * * X^{2}$ & 0,97 \\
\hline & Total & $Y=516,0-2,4864 * * X+0,004764 * * X^{2}$ & 0,96 \\
\hline \multirow{5}{*}{$\mathrm{Pb}$} & Raiz & $\mathrm{Y}=-822,0+310,63^{* *} \mathrm{X}-0,8316^{* *} \mathrm{X}^{2}$ & 0,86 \\
\hline & Caule & $Y=146,0+0,1184 * * X-0,001619^{* *} X^{2}$ & 0,85 \\
\hline & Folha & $\mathrm{Y}=8,6+30,5025 * * \mathrm{X}^{0,5}-1,4598^{* * X}$ & 0,77 \\
\hline & Parte Aérea & $\mathrm{Y}=158,0+2,1502 * * \mathrm{X}-0,005174 * * \mathrm{X}^{2}$ & 0,94 \\
\hline & Total & $\mathrm{Y}=-631,0+312,10^{* *} \mathrm{X}-0,8348^{* *} \mathrm{X}^{2}$ & 0,86 \\
\hline
\end{tabular}

** significativo a $1 \%$ de probabilidade, pelo teste $\mathrm{F}$.

A aplicação de $\mathrm{Pb}$ em solução nutritiva fez com que o conteúdo deste elemento aumentasse nas diferentes partes das plantas de ipê-roxo. Devido ao baixo teor de $\mathrm{Pb}$ no caule e na folha, o conteúdo deste elemento foi baixo nessas partes das plantas. $\mathrm{O}$ elevado teor de $\mathrm{Pb}$ na raiz (Quadro 2) fez com que o seu conteúdo fosse crescente até a dose de $187 \mu \mathrm{mol} \mathrm{Pb}$, apresentando o conteúdo máximo de $28.186 \mu \mathrm{g}$. Ao analisar a equação de regressão para o conteúdo total de $\mathrm{Pb}$, verifica-se que ela apresenta o ponto de máximo na dose de $187 \mu \mathrm{mol} \mathrm{Pb}$, dose esta exatamente a mesma para o máximo de conteúdo radicular, confirmando a pequena participação das demais partes das plantas.
Sabe-se que, em muitas espécies de plantas, o chumbo acumula-se preferencialmente nas raízes, tendo baixa translocação para a parte aérea (Eltrop et al., 1991; Bharti $\&$ Singh, 1993). Este confinamento às raízes, no entanto, provoca distúrbios fisiológicos, impedindo ou dificultando o pleno desenvolvimento das plantas, conforme observado por Paiva et al. (2000).

\section{CONCLUSÕES}

Os resultados obtidos a partir das mudas de ipê-roxo permitem concluir que:

R. Árvore, Viçosa-MG, v.27, n.2, p.151-158, 2003 
- a aplicação de $\mathrm{Pb}$ em solução nutritiva aumenta o teor de $\mathrm{P}$, principalmente na raiz. Os teores radiculares de $\mathrm{S}, \mathrm{Ca}$ e $\mathrm{Mg}$ não são afetados pela aplicação de $\mathrm{Pb}$;

- de modo geral, a aplicação de Pb provoca redução no teor de micronutrientes, exceção para o Zn, cujo teor não é afetado, independentemente da parte da planta;

- há redução no conteúdo de macro e de micronutrientes com a aplicação de doses crescentes de $\mathrm{Pb}$, em solução nutritiva; e

- em mudas de ipê-roxo o Pb acumula-se, preferencialmente, nas raízes.

\section{REFERÊNCIAS BIBLIOGRÁFICAS}

BAUMHARDT, G. R.; WELCH, L. F. Lead uptake and corn growth with soil-applied lead. Journal of

Environmental Quality, v. 1, n. 1, p. 92-94, 1972.

BERGMANN, W. Nutritional disorderes of plants: developments, visual and analytical diagnosis. New York: Gustav Fischer Verlang Jena, 1992. 741 p.

BHARTI, N.; SINGH, R. P. Growth and nitrate reduction by Sesamum indicum cv. PB-1 responds diferentially to lead. Phytochemistry, v. 33, n. 3, p. 531-534, 1993.

CLARK, R. B. Characterization of phosphatase of intact maize roots. Journal of Agriculture and Food Chemistry, v. 23, n. 3 , p. $458-460,1975$.

ELTROP, L. et al. Lead tolerance of Betula and Salix in the mining area of Mechernich/Germany. Plant and Soil, v. 131, n. 2 , p. $275-285,1991$.

FAQUIN, V. Nutrição mineral de plantas. Lavras: ESAL/ FAEPE. 1994. 227 p.

FODOR, F. et al. Lead uptake, distribution, and remobilization in cucumber. Journal of Plant Nutrition, v. 21, n. 7, p.1363-1373, 1998.

HUANG, J.W.; CUNNINGHAM, S.D. Lead phytoextraction: species variation in lead uptake and translocation. The New Phytologist, v. 134, n. 1, p. 75-84, 1996.

KABATA-PENDIAS, A.; PENDIAS, H. Trace elements in soils and plants. Boca Raton: CRC Press, 1984. 315 p.
KASTORI, R. et al. Effect of excess lead on sunflower growth and photosynthesis. Journal of Plant Nutrition, v. 21, n. 1 , p. $75-85,1998$.

LAGRIFFOUL, A. et al. Cadmium toxicity effects on growth, mineral and chlorophyll contents and activities of stress related enzymes in young maize plants (Zea mays L.). Plant and Soil, v. 200, n. 2, p. 241-250, 1998.

MALAVOLTA, E.; VITTI, G. C.; OLIVEIRA, S. A. Avaliação do estado nutricional das plantas: princípios e aplicações. 2.ed. Piracicaba: POTAFOS, 1997. 319 p.

MARSCHNER, P.; GODBOLD, D. L.; JENTSCHKE, G. Dynamics of lead accumulation in mycorrhizal and nonmycorrhizal Norway spruce (Picea abies (L.) Karst.). Plant and Soil, v. 178, n. 2, p. 239-245, 1996.

OBATA, H.; UMEBAYASHI, M. Effects of cadmium on mineral nutrient concentrations in plants differing tolerance to cadmium. Journal of Plant Nutrition, v. 20, n. 1, p. 97105, 1997.

PAIVA, H. N. Toxidez de Cd, Ni, Pb e Zn em mudas de cedro (Cedrela fissilis Vell.) e ipê-roxo (Tabebuia impetiginosa (Mart.) Standl.). 2000. 283 p. Tese (Doutorado em Fitotecnia) - Universidade Federal de Lavras, Lavras, 2000.

PAIVA, H. N.; CARVALHO, J. G.; SIQUEIRA, J. O. Efeito de $\mathrm{Cd}, \mathrm{Ni}, \mathrm{Pb}$ e $\mathrm{Zn}$ sobre mudas de cedro (Cedrela fissilis Vell.) e de ipê-roxo (Tabebuia impetiginosa (Mart.) Standley) em solução nutritiva. Revista Árvore, v. 24, n. 4, p. $369-378,2000$.

SIMON, L. Cadmium accumulation and distribution in sunflower plant. Journal of Plant Nutrition, v. 21, n. 2. p. 341-352, 1998.

SOARES, C. R. F. S. Toxidez de zinco, cobre, cádmio e chumbo para o eucalipto em solução nutritiva. 1999. 132 p. Dissertação (Mestrado em Solos e Nutrição de Plantas) - Universidade Federal de Lavras, Lavras, 1999.

WALKER, W. M.; MILLER, J. E.; HASSETT, J. J. Effect of lead and cadmium upon the calcium, magnesium, potassium, and phosphorus concentration in young corn plants. Soil Science, v. 124, n. 3, p. 145-151, 1977.

YANG, X. et al. Plant tolerance to nickel toxicity: II. Nickel effects on influx and transport of mineral nutrients in four plant species. Journal of Plant Nutrition, v. 19, n. 2, p. 265-279, 1996. 